\title{
Aplicação da ferramenta SWOT para avaliação das técnicas de dano ao choque térmico em materiais refratários
}

\section{(SWOT as a tool to evaluate the thermal shock damage technique of refractory materials)}

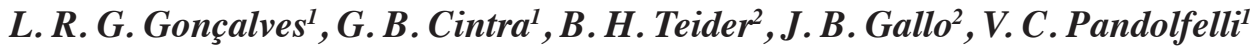 \\ ${ }^{1}$ Grupo de Engenharia de Microestrutura de Materiais - GEMM, Departamento de Engenharia de Materiais \\ Universidade Federal de S. Carlos, Rod. Washington Luiz, km 235, C.P. 676, S. Carlos, SP, 13565-905 \\ ${ }^{2}$ Alcoa Alumínio S.A, Departamento de Aplicações, Desenvolvimentos \& VPE, Rod. Poços - Andradas, km 10, \\ Poços de Caldas, $M G$ \\ lidiaufscar@yahoo.com.br,vicpando@ufscar.br
}

\begin{abstract}
Resumo
A literatura indica diversos tipos de ensaios e diferentes métodos de avaliação de dano por choque térmico. Adicionalmente sabe-se que, em determinadas aplicações, esse tipo de dano é um dos principais responsáveis pela degradação do revestimento refratário. Neste contexto e buscando a melhor forma de reproduzir as condições em serviço do revestimento refratário, o presente trabalho foi realizado visando analisar qual tipo de ensaio de choque térmico e método de avaliação do dano são os mais adequados para avaliar o desempenho destes materiais. Essa análise foi baseada em uma revisão bibliográfica e resultados obtidos em laboratório, em que se comparou e avaliou as vantagens e desvantagens dos métodos mais relevantes encontrados na literatura. Por fim, com o auxilio da ferramenta SWOT foi possível verificar se o método adotado pelos autores desse trabalho é adequado para auxiliar na seleção do refratário para aplicação em calcinadores de leito fluidizado.
\end{abstract}

Palavras-chave: choque térmico, refratários, SWOT.

\begin{abstract}
The literature shows different tests and techniques for evaluating the thermal shock damage. Additionally, it is known that, for certain applications, this type of wear mechanism is one of the main causes of fluid flash calciner refractory degradation. Within this context and in order to find the best way to reproduce the refractory lining service conditions, this work was carried out to point out which thermal shock test and damage assessment method are more suitable to evaluate these materials. This analysis was based on a literature review and laboratory experiments, comparing and evaluating the advantages and disadvantages of the most important methods available. Finally, with the support of the SWOT tool it was possible to evaluate whether the method adopted by the authors of this work is appropriated for helping the selection of the refractories for calciners application.
\end{abstract}

Keywords: thermal shock, refractories, SWOT.

\section{INTRODUÇÃO}

A análise SWOT, cujas siglas significam Strengths (forças), Weaknesses (fraquezas), Opportunities (oportunidades) e Threats (ameaças), é uma ferramenta muito utilizada por empresas na busca por orientações estratégicas. Seus pontos fortes e fracos são determinados por elementos internos, enquanto as oportunidades e riscos são ditados por forças externas, o que permite sistematizar todas as informações possíveis e, após uma análise cuidadosa, tomar uma decisão balanceada. No meio científico essa ferramenta pode ser uma alternativa eficaz para analisar se um método de medida adotado é adequado para a aplicação em questão.

$\mathrm{O}$ desgaste por choque térmico, assunto abordado no presente trabalho, é de grande importância técnica e econômica, já que em muitos casos, se trata de um dos principais responsáveis pela degradação do material refratário. Um exemplo prático que reproduz essas condições é o revestimento refratário de calcinadores de leito fluidizado usados na indústria de alumínio para a calcinação do hidróxido, transformando-o em óxido de alumínio. São conhecidos diversos tipos de ensaios de choque térmico. Abaixo segue uma breve descrição dos métodos mais relevantes destacando as respectivas vantagens e desvantagens.

\section{Forno com câmara dupla (Usiminas/GEMM)}

Este método utiliza um forno elétrico tubular contendo duas câmaras com temperaturas distintas e atmosfera controlada (Fig. 1). As amostras (150 mm x $30 \mathrm{~mm}$ x $30 \mathrm{~mm}$, pré-queimadas) são aquecidas em uma das câmaras e posteriormente são 
automaticamente transferidas para a outra com temperatura inferior, onde são resfriadas utilizando-se um fluxo de gás nitrogênio, gerando assim um gradiente de temperatura. No caso da Usiminas, são realizados um total de 6 ciclos (aquecimento a $1100{ }^{\circ} \mathrm{C}$ e resfriamento a $400{ }^{\circ} \mathrm{C}$, por exemplo), sendo que após 1, 3 e 6 ciclos são medidas as variações nas propriedades mecânicas, causadas pela degradação por choque térmico [1-3]. A avaliação do dano sofrido é realizada por medição do módulo elástico (MOE) e do módulo de ruptura a temperatura ambiente (MoR), além da avaliação visual.

Como vantagens esta técnica apresenta a independência do fator humano (método altamente automatizado), praticidade, ampla variedade de alternativas para o gradiente de temperatura, opção de adotar uma temperatura final que seja diferente da temperatura ambiente, possibilidade do controle de atmosfera (possibilita ensaios em materiais contendo carbono) e avaliação quantitativa do dano. As desvantagens que esse método apresenta é o fato de ainda não ser normalizado, a dificuldade de monitoramento da degradação em função do número de ciclos e a dificuldade de implementação (automatização, investimento inicial e dimensões). Sendo assim, se trata de um método muito prático e preciso que permite representar muito bem as variações de temperatura encontradas nas aplicações industriais. Porém, apresenta limitação na comparação de resultados (por ser um equipamento único) e o fato de ainda não estar normalizado pode resultar em uma difícil aceitação imediata.

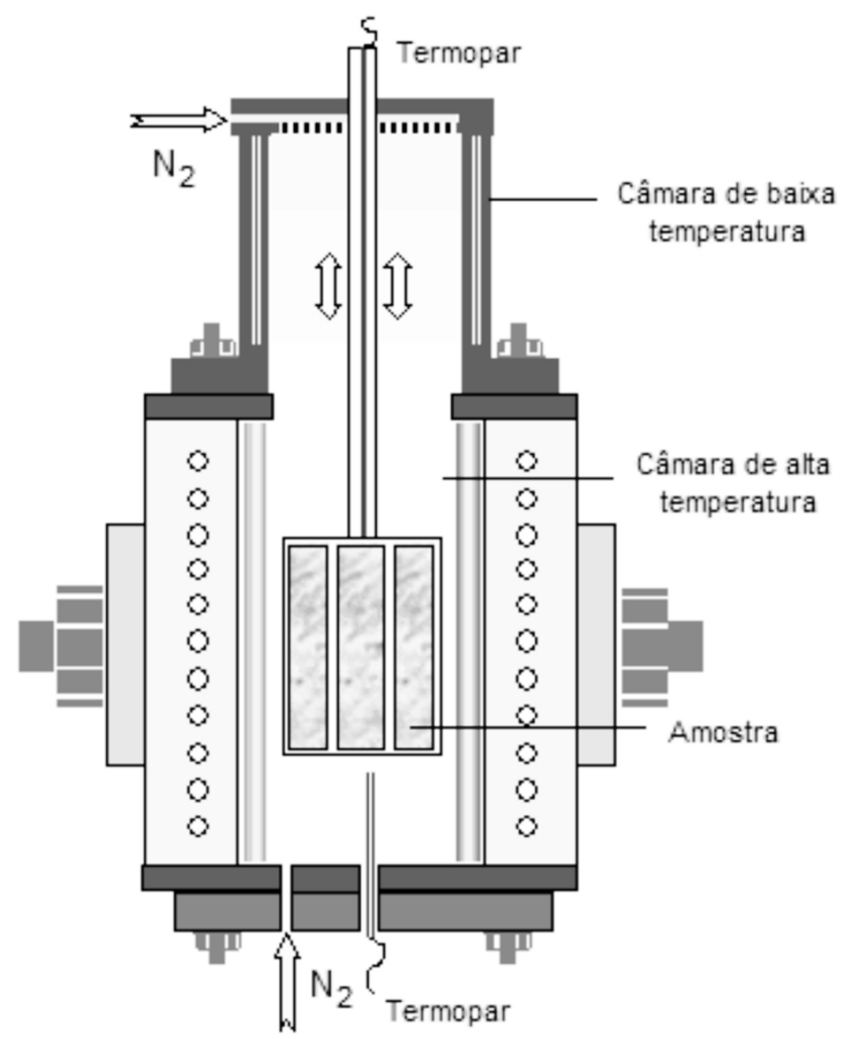

Figura 1: Forno de câmara dupla [1].

[Figure 1: Double chamber furnace [1].]

\section{Ribbon test}

Neste teste, as amostras $(228 \mathrm{~mm} \times 63,5 \mathrm{~mm} \times 63,5 \mathrm{~mm}$, separadas por $6 \mathrm{~mm}$ ) são alinhadas de forma que uma das suas faces seja submetida a uma chama contínua de gás quente, gerada por um maçarico (Fig. 2). A temperatura desta chama é rigorosamente controlada apresentando um desvio de no máximo $5{ }^{\circ} \mathrm{C}$. A distância entre as amostras e a chama de gás geralmente é de $127 \mathrm{~mm}$, podendo ser ajustada, se necessário.

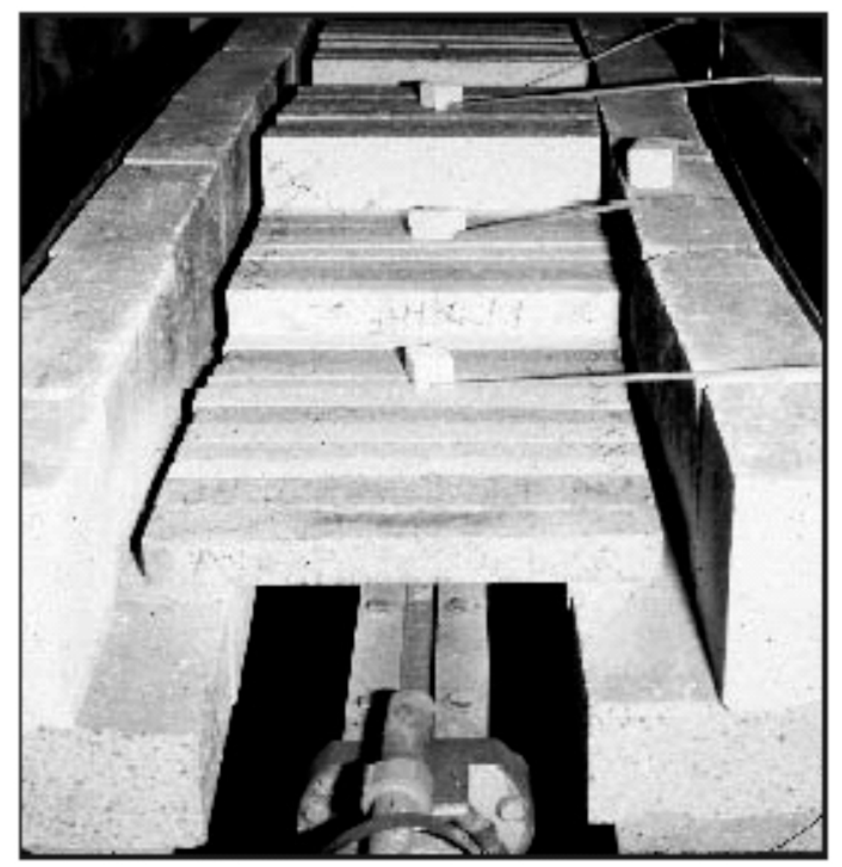

Figura 2: Equipamento para Ribbon test [3].

[Figure 2: Ribbon test equipment [3].]

O Ribbon test consiste em um método cíclico, no qual para o aquecimento são utilizados maçaricos e para o resfriamento são utilizados jatos de ar comprimido. Cada ciclo é de 30 min, sendo que 15 min correspondem ao aquecimento $(815$ a $1100{ }^{\circ} \mathrm{C}$ ) e o restante ao resfriamento, caracterizando uma variação brusca de temperatura. Os danos gerados são analisados por meio de variações das propriedades mecânicas que são medidas antes e depois dos ciclos térmicos [3-6]. O dano sofrido é avaliado pela medida do módulo elástico (MOE), resistência mecânica (MOR) e também pela avaliação visual. Apresenta como pontos positivos o fato de permitir a geração de gradientes de temperatura ao longo do corpo de prova, avaliação quantitativa do dano e é um método automatizado. Entretanto é um equipamento relativamente caro, com maior possibilidade de ser usado em escala industrial, além de necessitar de experiência técnica, gastos com energia, não possibilitar o controle da atmosfera (não adequado a materiais contendo carbono) e já ter sido eliminado das normas ASTM (2005). Atualmente é uma técnica alternativa para ser utilizada em empresas para a seleção e comparação de desempenho de diferentes materiais refratários (ex. ASTM C1100-88). 


\section{Panel spalling test}

Consiste basicamente em revestir internamente a porta de um forno (Fig. 3) com o material a ser ensaiado. $\mathrm{O}$ aquecimento é efetuado enquanto o forno encontra-se fechado e o resfriamento acontece com a abertura da porta. Trata-se de um ensaio que foi muito utilizado principalmente nos EUA e no Japão. Nos EUA as amostras (tijolos de 450 mm x $890 \mathrm{~mm}$ x $110 \mathrm{~mm}$ ) eram submetidas a 10 ciclos de 20 min cada, nos quais o aquecimento acontecia a $1400{ }^{\circ} \mathrm{C}$ por 10 min e em seguida as amostras eram resfriadas por spray de água. No Japão (amostras de 150 mm x 150 mm x 150 $\mathrm{mm})$, o aquecimento era realizado até $1700{ }^{\circ} \mathrm{C}$ durante $6 \mathrm{~h}$, seguido por um patamar de $8 \mathrm{~h}$ e um resfriamento a $400{ }^{\circ} \mathrm{C}$. Posteriormente o mesmo procedimento era repetido, porém com resfriamento a $200{ }^{\circ} \mathrm{C}$ [2].

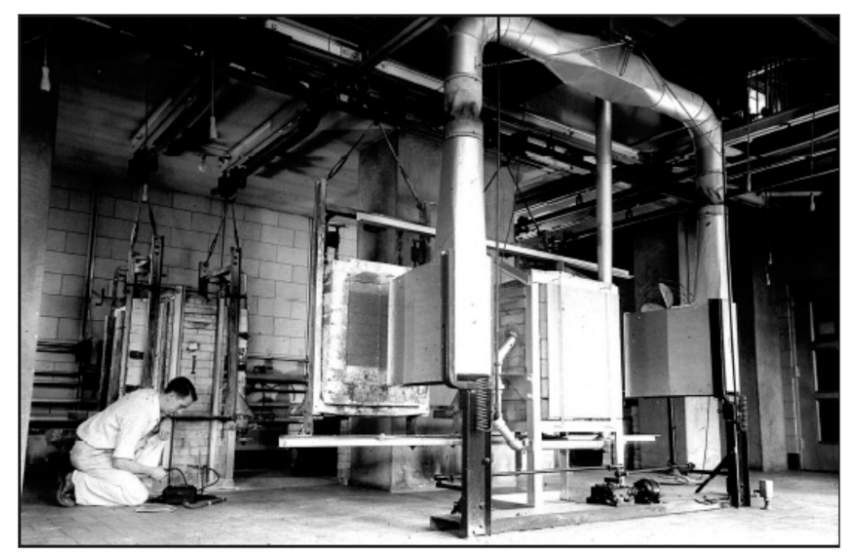

Figura 3: Equipamento para Panel Spalling test [3].

[Figure 3: Panel Spalling test equipment [3].]

A severidade do dano é proporcional à variação da massa em função do número de ciclos (EUA) ou pelo monitoramento do módulo elástico por emissão acústica (Japão). A principal vantagem apresentada por esse método era o fato de ser normalizado (ASTM C-38 e C-107), porém o mesmo já foi eliminado da ASTM em 1994. Já as desvantagens apresentadas são inúmeras, como o fato de ser impreciso na avaliação de refratários com maior resistência ao dano por CT, da impossibilidade de controle de atmosfera, da necessidade das amostras serem pré-queimadas, de apresentar um resfriamento muito lento (quando natural) e do provável dano ao forno. Atualmente se trata de um método pouco viável, pois além de ser indicado para materiais muito específicos, nem sempre o dano por choque térmico provoca perda de massa, o que torna inviável o método de medida em questão caso não se utilize à monitoração do dano por emissão acústica.

\section{Prism Spalling Test (adaptado pelo GEMM-UFSCar)}

Consiste em aquecer os corpos de prova utilizando fornos elétricos com temperatura entre 900 e $1200{ }^{\circ} \mathrm{C}$, por $15 \mathrm{~min}$, e em seguida resfriá-los por imersão em água (Alemanha e Japão), por exposição ao ar (EUA, Alemanha e
GEMM-UFSCar) ou por deposição em uma placa de metal refrigerada (Magnesita/Brasil), provocando assim uma variação brusca de temperatura [2,7]. A técnica utilizada no GEMM-UFSCar é baseada neste método (Fig. 4). Amostras com dimensões próximas a $150 \mathrm{~mm}$ x $25 \mathrm{~mm}$ x $25 \mathrm{~mm}$ são submetidas a uma variação de temperatura que é resultado de um aquecimento a aproximadamente $1000^{\circ} \mathrm{C}$ e resfriamento ao ar. Cada ciclo consiste de $15 \mathrm{~min}$ de aquecimento e 15 min de resfriamento. No total, cada corpo sofre 10 ciclagens térmicas. Para avaliar o dano sofrido é medido o módulo elástico inicial e após 3, 6 e 10 ciclos utilizando-se o método não destrutivo de ressonância de barras. Ao fim de 10 ciclos de choque térmico, mede-se a resistência mecânica residual. São várias as vantagens relacionadas a esse método, dentre elas o fato de ser normalizado (ASTM C-1171), de permitir o acompanhamento do dano sofrido (MOE versus número de ciclos), da avaliação do dano ser quantitativa (MOE versus ciclo / \%MOE versus número de ciclos), da praticidade e do custo relativamente baixo. Porém, também apresenta algumas desvantagens, como a limitação da temperatura do forno (1100-1200 ${ }^{\circ} \mathrm{C}$ ), a impossibilidade de controlar a atmosfera e o fator humano, uma vez que exige treinamento do operador. Portanto, este método possui fácil aplicação e permite a análise comparativa entre as composições de maneira eficiente.

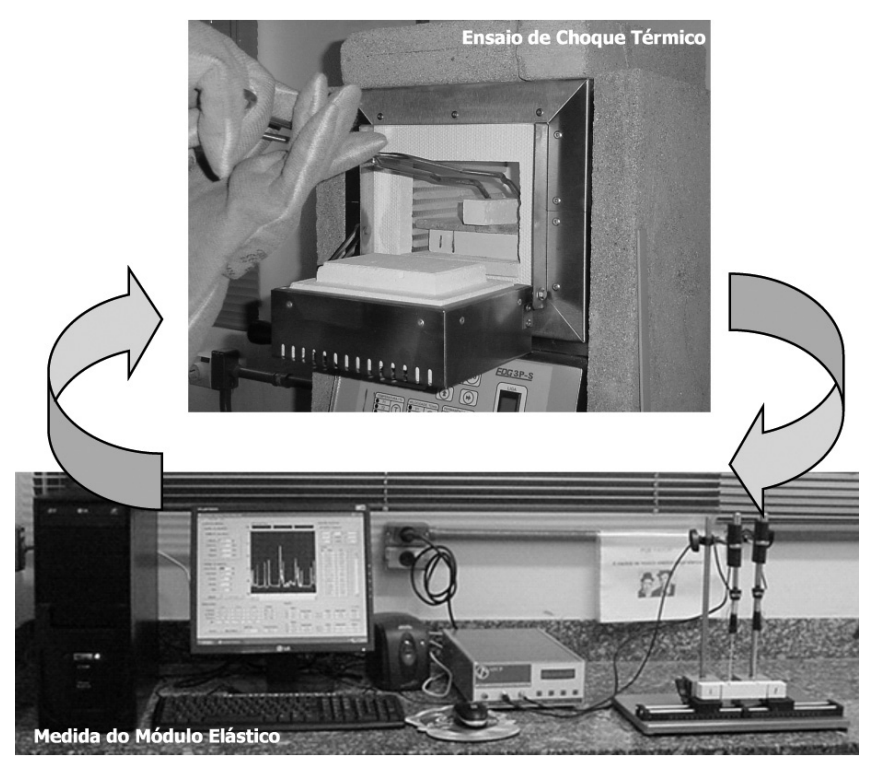

Figura 4: Equipamento utilizado no GEMM-UFSCar. [Figure 4: Equipment used by GEMM-UFSCar.]

\section{RESULTADOS E DISCUSSÃO}

O uso da ferramenta SWOT permite uma análise sistemática por meio da identificação das forças (S) e fraquezas $(\mathrm{W})$, bem como das oportunidades $(\mathrm{O})$ e ameaças (T), que quando interconectadas fornecem uma base sólida na tomada de uma decisão. No presente trabalho, a análise foi realizada com base no procedimento de medida de dano por choque térmico adotado pelo GEMM-UFSCar, 


\section{FATORES INTERNOS}

\begin{tabular}{|c|c|}
\hline & Strengths (Forcas) \\
\hline & 1. Normalizado (ASTM C-1171). \\
\hline & $\begin{array}{l}\text { 2. Permite acompanhamento do dano } \\
\text { sofrido (MOE vs } \mathrm{n}^{\circ} \text { de ciclos). }\end{array}$ \\
\hline $\begin{array}{l}\text { SWOT: método GEMM aplicado } \\
\text { no processo de seleção de }\end{array}$ & $\begin{array}{l}\text { 3. Praticidade. } \\
\text { 4. Custo relativamente baixo. } \\
\text { 5. Severa variação de temperatura. }\end{array}$ \\
\hline
\end{tabular}

\section{Weaknesses (Fraquezas)}

6. Limitação do forno $\left(1100{ }^{\circ} \mathrm{C}\right)$.

7. Impossibilidade de controlar a atmosfera.

8. Fator humano.

9. Corpos aquecidos como um todo.

10. Temperatura inferior necessariamente igual a temperatura ambiente.

11. Corpo de prova com volume restrito.

\begin{tabular}{|c|c|c|c|}
\hline \multirow[t]{2}{*}{ 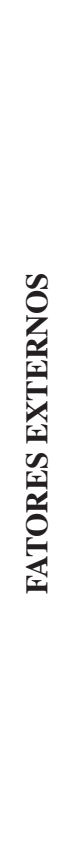 } & $\begin{array}{l}\text { Opportunities (Oportunidades) } \\
\text { A. Conhecimento das condições de } \\
\text { trabalho. } \\
\text { B. Classificar diferentes tipos de } \\
\text { refratário. }\end{array}$ & $\begin{array}{l}\text { S/O Implications } \\
\text { A/3-De uma forma simples é possível } \\
\text { representar bem as condições de } \\
\text { aplicação. (+) } \\
\text { B/1-Confere maior credibilidade } \\
\text { internacional e atende uma } \\
\text { necessidade global da empresa. (+) } \\
\text { B/2-Possibilidade de determinar um } \\
\text { ranking de desempenho, indicando } \\
\text { os refratários mais adequados. (+) }\end{array}$ & $\begin{array}{l}\text { W/O Implications } \\
\text { A/6-Dependendo das condições de uso, } \\
\text { a temperatura de aplicação pode ser } \\
\text { superior a máxima temperatura do } \\
\text { forno. (-) } \\
\text { A/7-Refratários usados em calcinadores não } \\
\text { necessitam de controle de atmosfera. } \\
(+) \\
\text { A/9-Diferença no perfil térmico nos testes } \\
\text { de laboratório e na aplicação. (-) } \\
\text { B/8-Fator humano pode reduzir a } \\
\text { precisão da medida e comprometer a } \\
\text { comparação. (-) } \\
\text { B/11-O tamanho da amostra não influencia } \\
\text { o ranking obtido desde que o volume } \\
\text { dos corpos de prova seja o mesmo } \\
\text { para todos os materiais testados. (+) }\end{array}$ \\
\hline & $\begin{array}{l}\text { Threats (Ameaças) } \\
\text { C. Temperatura de operação } \\
\text { variável. } \\
\text { D. Necessidade de reparos que } \\
\text { forçam resfriamento brusco. }\end{array}$ & $\begin{array}{l}\underline{\text { S/T Implications }} \\
\text { C/5 e D/5-As condições de ensaio } \\
\quad \begin{array}{l}\text { são mais críticas que as } \\
\text { condições de operação. (+) }\end{array}\end{array}$ & $\begin{array}{l}\frac{\text { W/T Implications }}{\text { C/10-Não representa as variações de }} \\
\text { temperatura causadas durante } \\
\text { diferentes campanhas. (-) }\end{array}$ \\
\hline
\end{tabular}

(+): vantagens./(-): desvantagens.

Figura 5: Análise SWOT aplicada ao método GEMM-UFSCar considerando a seleção de refratários para calcinador. [Figure 5: SWOT analyses applied to GEMM-UFSCar method for the selection of refractories for calciners.]

atribuindo como fatores internos todas as vantagens e desvantagens que esse método apresenta e que foram discutidas anteriormente, e os fatores externos como sendo as características da operação do calcinador para a indústria de alumínio. Assim, utilizando essa ferramenta, foi possível cruzar as informações do que se tem (método GEMM) com o que se deseja ter (selecionar o refratário mais adequado em relação ao CT para aplicar em calcinadores). De acordo com a análise SWOT (Fig. 5), o método apresenta importantes pontos positivos $(+)$, como o fato de ser normalizado, do método de medida permitir um acompanhamento do dano por meio de medidas de módulo elástico não destrutivas e por possibilitar a classificação de acordo com o desempenho, de forma pratica e confiável. Como o propósito desse trabalho é determinar o método mais adequado para a seleção de materiais, dentre os métodos existentes esse é extremamente vantajoso quando se trata de um processo classificatório. Observaram-se também algumas implicações que impactam negativamente no contexto da análise em questão (-). No entanto, no caso do item A/6 a limitação pode ser eliminada utilizando-se um forno que opere a temperaturas superiores, próximas a $1250{ }^{\circ} \mathrm{C}$. A implicação $\mathrm{B} / 8$ pode ser minimizada por meio de treinamentos das pessoas que irão operar o equipamento. Outra implicação negativa detectada foi a $\mathrm{A} / 9$, referente ao fato do corpo de prova não representar o mesmo perfil térmico da parede do calcinador, que apresenta uma face quente e outra fria. Nesse caso o método mais reprodutivo seria o Ribbon Test, porém como discutido anteriormente, esta técnica possui outros inconvenientes e, além disso, já foi excluída pela ASTM. E por fim, como ponto negativo tem a implicação $\mathrm{A} / 10$ que a princípio, indica que o método da Usiminas seria o mais adequado pelo fato de permitir uma maior possibilidade de variações de temperatura. No entanto, assim como justificado nas implicações $\mathrm{C} / 5$ e D/5, as condições de ensaio do método GEMM são mais criticas que as condições de operação, ou seja, o dano causado por um gradiente de temperatura cuja temperatura inferior é igual à temperatura ambiente é 
mais severo que aquele cuja temperatura inferior encontrase entre a temperatura ambiente e a temperatura superior. Portanto, é preciso ponderar a relevância desses fatores, determinando se os pontos negativos afetam ou não o processo de comparação.

\section{CONCLUSÃO}

A influência dos aspectos negativos apresentados pelo método analisado utilizando a ferramenta SWOT não compromete o propósito principal, que é escolher o material mais adequado para o revestimento de calcinadores de leito fluidizado, uma vez que os pontos positivos superam amplamente os negativos. Dessa forma o método GEMMUFSCar pode ser utilizado como ferramenta de medida do dano por choque térmico e considera-se, entre os testes disponíveis, esse método como o mais adequado e factível para esse fim.

\section{AGRADECIMENTOS}

Os autores agradecem a Alcoa pelo apoio técnico e ao CNPq pelo apoio financeiro.

\section{REFERENCIAS}

[1] M. Quintela, T. Melo, I. Lage, J. Rodrigues , V. C. Pandolfelli, Interceram - Refractories Manual (2003) 40-44. [2] C. E. Semler, "Thermal shock testing of refractories Revisited: Part I", Refractories Appl. News 13, 1 (2008) 18-22. [3] C. E. Semler, "Thermal shock testing of refractories Revisited: Part II”, Refractories Appl. News 13, 2 (2008) 18-24.

[4] ASTM C-1100, "Standard test method for Ribbon Thermal Shock testing of refractory materials", Annual Book of ASTM Standards 15.01 (1995) 295-298.

[5] C. E. Semler, T. H. Hawisher, "Evaluation of the thermal shock resistance of refractories using the Ribbon Test method", Am. Ceram. Soc. Bull. 59, 7 (1980) 732-738.

[6] C. E. Semler, T. H. Hawisher, R. C. Bradt, "Thermal shock of alumina refractories: damage-resistance parameters and the Ribbon Test", Am. Ceram. Soc. Bull. 60, 7 (1981) 724-729.

[7] ASTM C-1171, "Standard test method for quantitatively measuring the effect of thermal cycling on refractories", Annual Book of ASTM Standards 15.01 (1999) 316-318. (Rec. 25/06/2009, Ac. 28/08/2009) 